\title{
ON EXISTENCE OF POSITIVE SOLUTIONS OF NEUTRAL DIFFERENCE EQUATIONS*
}

\author{
J.H. SHEN, Z.C. WANG AND X.Z. QIAN
}

Abstract. Consider the neutral difference equation

$$
\triangle\left(x_{n}-c x_{n-m}\right)+p_{n} x_{n-k}=0, \quad n \geq N
$$

where $c$ and $p_{n}$ are real numbers, $k$ and $N$ are nonnegative integers, and $m$ is positive integer. We show that if

$$
\sum_{n=N}^{\infty}\left|p_{n}\right|<\infty
$$

then Eq. $(*)$ has a positive solution when $c \neq 1$. However, an interesting example is also given which shows that $(* *)$ does not imply that $(*)$ has a positive solution when $c=1$.

\section{Introduction}

For the last few years the oscillation and nonoscillation of solutions of delay difference equations are being extensively investigated [1-3,5-8], for a recent survey, we refer to [4]. In particular, the oscillation of solutions of the neutral difference equation

$$
\triangle\left(x_{n}-c x_{n-m}\right)+p_{n} x_{n-k}=0, \quad n \geq N
$$

have been intestigated in [9-10], where $c$ and $p_{n}$ are real numbers, $k$ and $N$ are nonnegative integers, and $m$ is positive integer. $\triangle$ denotes the forward difference operator $\Delta x_{n}=x_{n+1}-x_{n}$. However, the results for the existence of positive solutions of Eq.(1) are relatively scarce in the literature, we refer to $[10,11]$, see also Cyori and Ladas's book [4].

Received May 6,1993.

1991 Mathemetics Subject Classification. 39A10.

Key words and phrases. Neutral difference equations, positive soultions, oscillations.

* The project is supported by the National Natural Science Foundation of P.R. China. 
Our aim in this paper is to study the existence of positive solutions of Eq.(1). In section 2 we show that

$$
\sum_{n=N}^{\infty}\left|p_{n}\right|<\infty
$$

implies that Eq.(1) has a positive solution when $c \neq 1$. In section 3 , an interesting example is given to show that it is possible that Eq.(1) has no positive solutions under the hypothesis (2) when $c=1$.

Let $\rho=\max \{m, k\}$, by a solution of (1) we mean a sequence $\left\{x_{n}\right\}$ which is defined for $n \geq-\rho$ and which satisfies Eq.(1) for $n \geq N$. Clearly, if

$$
x_{n}=A_{n} \text { for } n=-\rho, \ldots,-1,0
$$

are given, then Eq.(1) has a unique solution satisfying the initial conditions (3). We assume throughout that $p_{n}$ cannot be eventualy identically zero. A nontrivial solution $\left\{x_{n}\right\}$ of (1) is said to be oscillatory if for every $N_{0} \geq N$ there exists a $n \geq N_{0}$ such that $x_{n} x_{n+1} \leq 0$, otherwise it is nonoscillatory.

\section{Positive solutions of Eq.(1)}

In this section we study the existence of positive solutions of Eq.(1) with $c \neq 1$. The main result in this section is the following theorem.

Theorem 1. Assume that (2) holds with $c \neq 1$, then Eq.(1) has a positive solution.

Proof. The proof of this theorem is rather too long and will be divided into five claims.

Claim 1. Show Theorem 1 for the case $0 \leq c<1$.

Indeed, choose a positive integer $N_{0} \geq N$ sufficiently large such that $N_{0}-\rho \geq N$ and

$$
\sum_{n=N_{0}}^{\infty}\left|p_{n}\right| \leq \frac{1-c}{4}
$$

Consider the Banach Space $l_{\infty}^{N}$ of all real sequences $x=\left\{x_{n}\right\}$ where $n \geq N$ with sup norm $\|x\|=\sup _{n \geq N}\left|x_{n}\right|$. We define a subset $S$ in $l_{\infty}^{N}$ as

$$
S=\left\{x \in l_{\infty}^{N}: 2(1-c) / 3 \leq x_{n} \leq 4 / 3, n \geq N\right\}
$$

Then $S$ is a bounded, closed and convex subset of $l_{\infty}^{N}$. Now we define an operator $T: S \rightarrow l_{\infty}^{N}$. For $x \in S$,

$$
T x_{n}= \begin{cases}1-c+c x_{n-m}+\sum_{i=n}^{\infty} p_{i} x_{i-k}, & n \geq N_{0}, \\ T x_{N_{0}} & N \leq n \leq N_{0} .\end{cases}
$$


Clearly, $T$ is continuous. For every $x=\left\{x_{n}\right\} \in S, n \geq N_{0}$, we have

$$
\begin{aligned}
T x_{n} & \leq 1-c+\frac{4}{3} c+\frac{4}{3} \sum_{i=n}^{\infty}\left|p_{i}\right| \\
& \leq 1-c+\frac{4}{3} c+\frac{4}{3} \cdot \frac{1-c}{4}=\frac{4}{3}
\end{aligned}
$$

and

$$
T x_{n} \geq 1-c+\frac{4}{3} \cdot \frac{c-1}{4}=\frac{2(1-c)}{3}
$$

Hence, $2(1-c) / 3 \leq T x_{n} \leq 4 / 3$ for $n \geq N$, and so $T S \subset S$.

Now we will show that $T$ is a contraction mapping on $S$. In fact, for any $x, y \in S$ and $n \geq N_{0}$, we have

$$
\begin{aligned}
\left|T x_{n}-T y_{n}\right| & \leq c\left|x_{n-m}-y_{n-m}\right|+\sum_{i=n}^{\infty}\left|p_{i}\right| \cdot\left|x_{i-k}-y_{i-k}\right| \\
& \leq\left(c+\frac{1-c}{4}\right)\|x-y\|=\frac{1+3 c}{4}\|x-y\| .
\end{aligned}
$$

It follows that

$$
\|T x-T y\| \leq \frac{1+3 c}{4}\|x-y\|
$$

Since $0<(1+3 c) / 4<1$, we see that $T$ is a contraction on $S$. Therefore, by the Banach contraction princile, $T$ has a fixed point $x \in S$, i.e., $T x=x$. It is easy to see that $x=\left\{x_{n}\right\}$ is a positive solution of Eq.(1) and so the proof of Claim 1 is complete.

Claim 2. Theorem 1 holds for the case $c>1$. Let $N_{0} \geq N$ be such that $N_{0}+m-$ $k \geq N$ and

$$
\sum_{n=N_{0}+m}^{\infty}\left|p_{n}\right| \leq-\frac{1-c}{4}
$$

Consider the Banach Space $l_{\infty}^{N}$ as in the proof of Claim 1. Set

$$
S=\left\{x \in l_{\infty}^{N}: c / 2 \leq x_{n} \leq 2 c \text { for } n \geq N\right\}
$$

Then $S$ is a bounded, closed and convex subset of $l_{\infty}^{N}$. Define a mapping $T: S \rightarrow l_{\infty}^{N}$ as following

$$
T x_{n}= \begin{cases}c-1+\frac{1}{c} x_{n+m}-\frac{1}{c} \cdot \sum_{i=n+m}^{\infty} p_{i} x_{i-k}, & n \geq N_{0}, \\ T x_{N_{0}}, & N \leq n \leq N_{0} .\end{cases}
$$

Clearly, $T$ is continuous. For every $x=\left\{x_{n}\right\} \in S$ and $n \geq N_{0}$, we have

$$
\begin{aligned}
T x_{n} & \leq c-1+\frac{2 c}{c}+\frac{1}{c} 2 c \sum_{i=n+m}^{\infty}\left|p_{i}\right| \\
& \leq c-1+2+2 \frac{c-1}{4}=\frac{3 c+1}{2}<2 c
\end{aligned}
$$


and

$$
T x_{n} \geq c-1+\frac{1}{c} \cdot \frac{c}{2}-\frac{1}{c} \cdot 2 c \cdot \frac{c-1}{4}=\frac{c}{2}, \text { for } n \geq N_{0}
$$

Hence, $c / 2 \leq T x_{n} \leq 2 c$ for $n \geq N$, and so $T S \subset S$. Now by a proof similar to the proof of Claim 1, we see that, for any $x, y \in S$,

$$
\|T x-T y\| \leq \frac{3+c}{4 c} \cdot\|x-y\|
$$

Since $0<(3+c) / 4 c<1$, it follows that $T$ is a contraction on $S$. Therefore, by the Banach Contraction Princile, $T$ has a fixed point $x=\left\{x_{n}\right\} \in S$. It is easy to see that this $x$ is a positive solution of Eq.(1) and the proof of Claim 2 is complete.

Claim 3. Prove Theorem 1 for the case $-1<c<0$.

Let $N_{0} \geq N$ be such that $N_{0}-\rho \geq N$ and

$$
\sum_{n=N_{0}}^{\infty}\left|p_{n}\right| \leq \frac{1+c}{4}
$$

Let $l_{\infty}^{N}$ be defined as in the proof of Claim 1. Clearly, the set

$$
S=\left\{x \in l_{\infty}^{N}: 2(1+c) \leq x_{n} \leq 4 \text { for } n \geq N\right\}
$$

is a bounded, closed and convex subset of $l_{\infty}^{N}$. Define $T: S \rightarrow l_{\infty}^{N}$

$$
T x_{n}= \begin{cases}3-c+c x_{n-m}+\sum_{i=n}^{\infty} p_{i} x_{i-k}, & n \geq N_{0} \\ T x_{N_{0}}, & N \leq n \leq N_{0}\end{cases}
$$

Clearly, $T$ is continuous. It is easy to see that $T$ maps $S$ into itself, and for any $x, y \in S$,

$$
\|T x-T y\| \leq \frac{1-3 c}{4} \cdot\|x-y\|
$$

As $0<(1-3 c) / 4<1$, the Banach Contraction Priciple can be applied to obtain a fixed point $x=\left\{x_{n}\right\}$ of $T$. It is easy to see that this $\left\{x_{n}\right\}$ is a positive solution of Eq.(1). This completes the proof of Claim 3.

Claim 4. Theorem 1 holds for the case $c=-1$.

Indeed, let $N_{0} \geq N$ be such that $N_{0}+m-k \geq N$ and

$$
\sum_{n=N_{0}+m}^{\infty}\left|p_{n}\right| \leq \frac{1}{4}
$$

Let $l_{\infty}^{N}$ be defined as in the proof of Claim 1.Then

$$
S=\left\{x \in l_{\infty}^{N}: 2 \leq x_{n} \leq 4 \text { for } n \geq N\right\}
$$


is a bounded, closed and convex subset of $l_{\infty}^{N}$. Now we deifne a mapping as following

$$
T x_{n}= \begin{cases}3+\sum_{j=1}^{\infty} \cdot \sum_{i=n+(2 j-1) m}^{n+2 m j-1} p_{i} x_{i-k}, & n \geq N_{0} \\ T x_{N_{0}}, & N \leq n \leq N_{0}\end{cases}
$$

Since, for any $x=\left\{x_{n}\right\} \in S$ and $n \geq N_{0}$,

$$
\begin{aligned}
T x_{n} & \leq 3+\sum_{j=1}^{\infty} \sum_{i=n+(2 j-1) m}^{n+2 m j-1} 4\left|p_{i}\right| \\
& \leq 3+4 \cdot \sum_{i=N_{0}+m}^{\infty}\left|p_{i}\right| \\
& \leq 3+4 \cdot \frac{1}{4}=4
\end{aligned}
$$

and.

$$
\begin{aligned}
T x_{n} & \geq 3-4 \cdot \sum_{j=1}^{\infty} \sum_{i=n+(2 j-1) m}^{n+2 m j-1}\left|p_{i}\right| \geq 3-4 \cdot \sum_{i=N_{0}+m}^{\infty}\left|p_{i}\right| \\
& \geq 3-4 \cdot \frac{1}{4}=2
\end{aligned}
$$

it follows that $T$ maps $S$ into $S$. It is also not difficult to see that for any $x, y \in S$ we have

$$
\|T x-T y\| \leq \frac{1}{4} \cdot\|x-y\|
$$

Therefore, the Banach Contraction Princile can be applied to obtain a fixd point $x \in S$ of $T$, that is,

$$
x_{n}= \begin{cases}3+\sum_{j=1}^{\infty} \sum_{i=n+(2 j-1) m}^{n+2 m j-1} p_{i} x_{i-k}, & n \geq N_{0} \\ x_{N_{0}}, & N \leq n \leq N_{0}\end{cases}
$$

It follows that

$$
\begin{aligned}
x_{n}+x_{n-m} & =6+\sum_{j=1}^{\infty}\left[\sum_{i=n-m+(2 j-1) m}^{n-m+2 m j-1} p_{i} x_{i-k}+\sum_{i=n+(2 j-1) m}^{n+2 m j-1} p_{i} x_{i-k}\right] \\
& =6+\sum_{i=n}^{\infty} p_{i} x_{i-k}, \text { for } n \geq N_{0}+m
\end{aligned}
$$

From this we see that $x=\left\{x_{n}\right\}$ is a positive soluton of Eq.(1) on $n \geq N_{0}+m$, and so the proof of Claim 4 is complete.

Claim 5. Complete the proof of Theorem 1 when $c<-1$. 
Let $N_{0} \geq N$ be such that $N_{0}+m-k \geq N$ and

$$
\sum_{n=N_{0}+m}^{\infty}\left|p_{n}\right| \leq \frac{-c-1}{4}
$$

Let $l_{\infty}^{N}$ be defined as in the proof of Claim 1 and let

$$
S=\left\{x \in l_{\infty}^{N}:-2(c+1) \leq x_{n} \leq-4 c, n \geq N\right\}
$$

Clearly, $S$ is a bounded, closed and convex subset of $l_{\infty}^{N}$. Define a mapping $T$ on $S$ as following

$$
T x_{n}= \begin{cases}-3 c+1+\frac{1}{c} x_{n+m}+\sum_{i=n+m}^{\infty} p_{i} x_{i-k}, & n \geq N_{0} \\ T x_{N_{0}}, & N \leq n \leq N_{0}\end{cases}
$$

By an argument similar to that in the proof of Claim 2 we can easily show that the all hypotheses of the Banach Contraction Principle are satisfied. Therefore, $T$ has a fixed point $x=\left\{x_{n}\right\} \in S$. It is easy to see that this $\left\{x_{n}\right\}$ is a positive solution of Eq.(1) for $n \geq N_{0}+m$ and the proof of Claim 5 is complete.

Combining Claim 1-5, we see that the proof of Theorem 1 is complete.

\section{An example}

The aim in the section is to show by the folloing example that Theorem 1 does not hold when $c=1$.

Example 1. Consider the neutral difference equation

$$
\Delta\left(x_{n}-x_{n-1}\right)+\frac{1}{n \ln ^{2} n} x_{n-1}=0, n \geq 2
$$

Here $m=k=1, c=1$, and $p_{n}=1 / n \ln ^{2} n$, Since

$$
\sum_{n=2}^{\infty} p_{n}=\sum_{n=2}^{\infty} \frac{1}{n \ln ^{2} n}<\infty
$$

it follows that (2) holds. Next we will prove that Eq.(4) has no positive solutions. Otherwise, assume that (4) has a positive solution $=\left\{x_{n}\right\}$ satisfying $x_{n-1}>0, n \geq$ $N_{0} \geq 2$, for some $N_{0} \geq 2$. Set

$$
z_{n}=x_{n}-x_{n-1}, \text { for } n \geq N_{0}
$$

Then by (4) we have

$$
\Delta z_{n}=-\frac{1}{n \ln ^{2} n} x_{n-1}<0 \quad \text { for } \quad n \geq N_{0}
$$


We consider the following two possible cases:

(i) If $z_{n}$ is eventually negative, then by (5) we see that there exist $\alpha>0$ and positive integer $N_{1} \geq N_{0}$, such that

$$
z_{n} \leq-\alpha \text { for } n \geq N_{1}
$$

This is

$$
x_{n} \leq-\alpha+x_{n-1}, \text { for } n \geq N_{1}
$$

By using the Induction Principle, we get

$$
x_{n+N_{1}} \leq-n \alpha+x_{N_{1}} \rightarrow-\infty \text { as } n \rightarrow \infty
$$

which contradicts the positivity of $\left\{x_{n}\right\}$.

(ii) If $z_{n}$ is eventually positive, then there exists positive integer $N_{2} \geq N_{1}$ such that $z_{n}>0$ for $n \geq N_{2}$. Now we let

$$
\lim _{n \rightarrow \infty} z_{n}=\beta \in[0, \infty)
$$

and $\operatorname{sum}(5)$ from $n \geq N_{2}$ to $\infty$, we have

$$
\beta-z_{n}+\sum_{k=n}^{\infty} \frac{1}{k \ln ^{2} k} x_{k-1}=0
$$

this implies that

$$
\sum_{n=N_{2}}^{\infty} \frac{1}{n \ln ^{2} n} x_{n-1}<\infty
$$

On the other hand, since $x_{n}-x_{n-1}>0$, for $n \geq N_{2}$, it follows that there exists a positive constant $M$ such that $x_{n} \geq M$ for $n \geq N_{2}$, Substituting this into (6) we find that

$$
\begin{aligned}
z_{n} & \geq \beta+M \sum_{k=n}^{\infty} \frac{1}{k \ln ^{2} k} \geq \beta+M \int_{n}^{\infty} \frac{1}{t \ln ^{2} t} d t \\
& =\beta+\frac{M}{\ln n}, \text { for } n \geq N_{2}+1
\end{aligned}
$$

that is,

$$
x_{n} \geq x_{n-1}+\frac{M}{\ln n}, \text { for } n \geq N_{2}+1
$$

It follows that

$$
\begin{aligned}
x_{n} & \geq M\left[\frac{1}{\ln n}+\frac{1}{\ln (n-1)}+\cdots+\frac{1}{\ln \left(n+1-\left(n-N_{2}\right)\right)}\right]+x_{N_{2}} \\
& \geq M \frac{n-N_{2}}{\ln n}, \text { for } n \geq N_{2}+1
\end{aligned}
$$


Hence, we find that

$$
\frac{1}{n \ln ^{2} n} x_{n-1} \geq M \frac{n-N_{2}-1}{n \ln ^{2} n \ln (n-1)} \text { for } n \geq N_{2}+2
$$

As $\sum_{n=N_{2}}^{\infty} \frac{n-N_{2}-1}{n \ln ^{2} n \ln (n-1)}=\infty$, it follows from (7) that

$$
\sum_{n=N_{2}}^{\infty} \frac{1}{n \ln ^{2} n} x_{n-1}=\infty
$$

This contradiction shows that the case (ii) is also impossible. The proof which Eq.(4) has no positive solutions is complete.

The following example 2 shows that it is also possible that Eq.(1) has a positive solution when (2) holds and $c=1$.

Example 2. Consider the neutral difference equation

$$
\triangle\left(x_{n}-x_{n-1}\right)+p_{n} x_{n-1}=0, n \geq 1
$$

Here $p_{n}=\frac{1}{n(n+1)}\left(\sum_{i=1}^{n-1} \frac{1}{i}\right)^{-1}$ It is obvious that (2) holds, and this equation has a positive solution $x_{n}=\sum_{i=1}^{n} 1 / i, n=1,2, \cdots$

Remark. Combining the Theorem 1 and Example 1, we know that

$$
\sum_{n=N}^{\infty}\left|p_{n}\right|=\infty
$$

is an necessary condition for the oscillation of all solutions of Eq.(1) when $c \neq 1$.

\section{References}

[1] G. Ladas, Recent developments in the oscillations of delay difference equations, in Differential Equations: Stability and Control, Dekker, New York, 1990.

[2] L.H. Erbe and B.G. Zhang, "Oscillation of discrete analogues of delay equations," Differential and Integral Equations, 2, No.3 (1989), 300-309.

[3] G. Ladas, "Explicit conditions for the oscillation of difference equations," J. Math. Anal. Appl., 153(1990), 276-287.

[4] I. Györi and G. Ladas, Oscillation Theory of Delay Differential Equations with Applications, Clarendon Press, Oxford, 1991.

[5] G. Ladas et al., "Necessary and sufficient conditions for the oscillation of difference equations," Libertas Math., 9(1989), 121-.

[6] G. Ladas et al., "Sharp conditions for the oscillations of delay difference equations," J. Appl. Math. Simulation, in press.

[7] L.H. Erbe and B.G. Zhang, "Oscillation for first order linear differential equations with deviation arguments," Differential and Integral Equations,1988. 
[8] Jurang Yan and Chuanxi Qian, "Oscillation and comparison results for delay difference equations," J. Math. Anal. Appl., 165(1992), 346-357.

[9] D.A. Georgiou et al., "Oscillations of neutral difference equations," Appl. Anal., Nos. 3-4, 33(1989), 24.3-253.

[10] B.S. Lalli et al., "On the oscillation of solutions and existence of positive solutions of neutral difference equations," J. Math. Anal. Appl., 158(1991), 213-233.

[11] B.S. Lalli and B.G. Zhang, "On existence of positive solutions and bounded oscillations for neutral difference equations," J. Math. Anal. Appl., 166 (1992), 272-287.

Department of Applied Mathematics, Hunan University, Changsha, Hunan 410082, China. 